\title{
BMJ Open Risk factors for gastric cancer and related serological levels in Fujian, China: hospital-based case-control study
}

\author{
Ping Yuan (D , ,,2 Lan Lin, ${ }^{3,4}$ Kuicheng Zheng, ${ }^{1,2}$ Wen Wang, ${ }^{5}$ Sihan Wu, ${ }^{3}$ \\ Liangxiang Huang, ${ }^{4}$ Bingshan $\mathrm{Wu},{ }^{1}$ Tiehui Chen, ${ }^{3}$ Xiaoqing $\mathrm{Li}^{3}{ }^{3}$ Lin $\mathrm{Cai}^{2}$
}

To cite: Yuan $P$, Lin L, Zheng $\mathrm{K}$, et al. Risk factors for gastric cancer and related serological levels in Fujian, China: hospitalbased case-control study. BMJ Open

2020;10:e042341. doi:10.1136/ bmjopen-2020-042341

- Prepublication history for this paper is available online. To view these files, please visit the journal online (http://dx.doi. org/10.1136/bmjopen-2020042341).

PY and LL contributed equally.

Received 04 July 2020

Revised 11 August 2020 Accepted 12 August 2020

Check for updates

(c) Author(s) (or their employer(s)) 2020. Re-use permitted under CC BY-NC. No commercial re-use. See rights and permissions. Published by BMJ.

For numbered affiliations see end of article.

Correspondence to

Kuicheng Zheng;

zkcfjcdc@sina.com

\section{ABSTRACT}

Objective To explore the relationships between gastric cancer and serum pepsinogen I (PG I), PG II, PG I/II ratio, gastrin 17 (G-17) and Helicobacter pylori infection, and to investigate dietary and lifestyle risk factors for gastric cancer in Fujian Province, China.

Design A hospital-based, 1:1 matched case-control study.

Setting Patients with newly diagnosed gastric cancer were recruited from the Fujian Provincial Hospital and the No. 900 Hospital of the Joint Support Force of the Chinese People's Liberation Army between July 2014 and December 2016.

Participants A total of 180 pairs of patients with gastric cancer and control subjects were recruited in the study, including 134 (74.4\%) male pairs and 46 (25.6\%) female pairs.

Investigation and analysis measures Serological indicators were tested with ELISA kits. Dietary, lifestyle and psychological factors were investigated through face-toface questionnaire. Relationships between gastric cancer and these influencing factors were examined by $\mathrm{X}^{2}$ test and conditional logistic regression.

Results Serum PG II and G-17 levels and H. pylori infection rate were higher in patients with gastric cancer than in control subjects $(p<0.05)$, while $P G I / I I$ ratio was lower in patients with gastric cancer $(p<0.05)$. Serum G-17 levels were higher in patients with corpus gastric cancer than in patients with antral gastric cancer $(p<0.05)$. Serum PG II levels were higher in patients with advanced gastric cancer than in patients with early-stage cancer $(p<0.05)$, however, $P G \mathrm{I} / \mathrm{II}$ ratio was lower in patients with advanced-stage gastric cancer than in patients with earlystage cancer $(p<0.05)$. Eating hot food $(0 R=2.32)$, eating pickled vegetables $(\mathrm{OR}=4.05)$ and often feel troubled $(O R=2.21)$ were found to significantly increase the risk of gastric cancer (all $p<0.05$ ), while consuming onion or garlic $(\mathrm{OR}=0.35)$, drinking tea $(\mathrm{OR}=0.26)$, eating fresh fruits $(\mathrm{OR}=0.55)$, and high serum $\mathrm{PG}$ I $(\mathrm{OR}=0.99)$ or $\mathrm{PG} \mathrm{I/II}$ ratio $(O R=0.73)$ were found to be protective against gastric cancer.

Conclusion Study results showed that serum PG, G-17 and $H$. pylori antibodies could be useful indicators for early diagnosis of gastric cancer. Increase in serum G-17 level might indicate the location of gastric cancer. Increase in serum PG II level and decrease in PG I/II ratio might
Strengths and limitations of this study

- Fujian Province, high in gastric cancer incidences, is an important research site for exploring the aetiologies of gastric cancer.

- This study was one of the few studies to use serum indicators as independent variables to analyse the risk factors for gastric cancer.

- Strict quality control was conducted in the selection of new cases to ensure comparable results.

- However, as a case-control study, recall bias was inevitable and trial studies are required for more accurate results.

- Sample size of this study was not large enough, further studies will recruit more subjects.

imply the clinical stage. Eating hot food, eating pickled vegetables and often feel troubled may be risk factors for gastric cancer, while eating fresh fruits, eating onion or garlic, and drinking tea may be protective factors against the disease.

\section{INTRODUCTION}

Gastric cancer is a common malignancy in the gastric mucosa and gastric glands in the digestive tract and accounts for a high proportion of cancer deaths in China, especially in Fujian Province. According to the World Cancer Report published by the WHO in 2018, ${ }^{1}$ there were 1033071 new cases of gastric cancer (accounting for $5.7 \%$ of all cancer incidences) and 782685 deaths from it (accounting for $8.2 \%$ of all cancer deaths) worldwide. Gastric cancer has high disease burdens, ${ }^{2}$ ranking second only to lung cancer in terms of the number of deaths. ${ }^{1}$ In China, there were 456124 new cases of gastric cancer in 2018 , accounting for $44.1 \%$ of global incidence. ${ }^{3}$ In Fujian Province, located in southeast China, gastric cancer accounted for $12.5 \%$ of all cancer incidences in $2014 .{ }^{4}$ Risk factors for gastric cancer such as dietary, lifestyle and 
psychological factors are different across China. ${ }^{5-10}$ The overall objectives of this study were to explore the relationship between gastric cancer and serum pepsinogen I (PG I), PG II, PG I/II ratio, gastrin-17 (G-17) and Helicobacter pylori infection, and to investigate the risk factors for gastric cancer in Fujian.

\section{METHODS}

\section{Study design}

This was a hospital-based, 1:1 matched case-control study, performed in accordance to the Declaration of Helsinki of the World Medical Association. Informed consents were obtained from all recruited subjects. Subjects' dietary and lifestyle data were obtained through face-to-face interviews by trained investigators, and blood samples were collected for the test of serum markers.

\section{Case and control groups}

From July 2014 to December 2016, patients with newly diagnosed gastric cancer were recruited from the Fujian Provincial Hospital and the No. 900 Hospital of the Joint Support Force of the Chinese People's Liberation Army. Control subjects were recruited simultaneously from the Medical Examination Center at the same hospital. Patients with gastritis and control subjects were 1:1 matched according to gender and similar age within 3 years. Inclusion criteria for the gastric cancer group were: newly diagnosed of gastric cancer by gastroscopy and pathology, with no medication history of antibiotics, proton pump inhibitors or $\mathrm{H}$ receptor antagonists. Patients with prior surgeries or chemotherapies were also excluded. Inclusion criteria for the control group were: no diagnosis or symptoms of chronic stomach diseases, and no mental retardation or emotional blockages. All subjects had lived in Fujian Province for more than 10 years.

\section{Risk factor survey}

Data of risk factors were collected by trained investigators through face-to-face interviews with the subjects, using a consolidated questionnaire. The survey contents included general information, dietary habits (ie, breakfast consumption, meal duration, intake frequencies of onion or garlic, spicy food, hot food, green vegetables, fresh fruit, dairy, pickled vegetables and fish sauce), lifestyle habits (ie, smoking, alcohol consumption and tea consumption) and psychological factors (ie, personality type, adaptability, feels about life and interpersonal skills).

\section{Serum sampling}

A total of $5 \mathrm{~mL}$ of fasting venous blood was collected from each subject and was centrifuged at $3000 \mathrm{r} / \mathrm{min}$ for $10 \mathrm{~min}$ to extract the serum, which was then stored at $-80^{\circ} \mathrm{C}$ for further testing. Serum PG I, PG II and G-17 levels were tested using ELISA kits from BIOHIT, Finland (batch numbers of 19PA1505, 21PB1508 and 19GC1506, respectively). Antibodies to $H$. pylori were assessed using ELISA kits from the AI Kang Company of China (lot number 201510087).

\section{Definitions and variables}

Dietary intake frequencies were classified as: never or seldom consumption (no more than once per week), occasional consumption (two to six times per week) and regular consumption (seven or more times per week). Smokers were defined as subjects who had smoked more than 100 cigarettes overall. Alcohol consumers were defined as subjects who had been drinking any alcohol at least once a week for more than 6 months. Tea consumers were defined as subjects who had been drinking any tea at least once a week for more than 6 months.

According to the calculated cut-off value, an optical density $(\mathrm{OD})$ value of the sample $>1.1 \times$ the cut-off value was considered $H$. pylori positive, while an OD $<0.9 \times$ the cut-off value was considered $H$. pylori negative.

\section{Statistical analysis}

Data were inputted by the double-entry method and tested for consistency using Epidata V.3.1. The SPSS V.24.0 software package was used for conducting $\mathrm{X}^{2}$ tests on the demographic information, and for conducting the single and multivariate conditional logistic regression analyses on the other information to determine the OR and $95 \%$ CI. Test significance level was set at 0.05 .

\section{Patient and public involvement}

Dr Kazuo Aoki, a Japanese expert, was involved in designing the study due to the growing concern among people with gastric cancer both in Japan and China. This study was undertaken in the two hospitals with the firstclass at grade 3 in Fujian province in order to ensure the quality of research and access to adequate and widely sourced cases. The findings of this study will be published to inform the public about the risk factors for gastric cancer and some of the inspection results were fed back to the subjects through the form of inspection report.

\section{RESULTS}

\section{Demographic information}

A total of 180 pairs of patients with gastric cancer and control subjects were recruited in this study, of which 134 pairs $(74.4 \%)$ were male subjects and 46 pairs $(25.6 \%)$ were female subjects. The average ages of the cancer group and the control group were $61.0 \pm 10.8$ and $60.1 \pm 10.9$ years, respectively. The two groups were not significantly different in age, marital status, education or labour intensity (all $\mathrm{p} \geq 0.05$ ), however the occupation compositions were different.

\section{Dietary habits, lifestyles and personality traits}

As shown in table 1, there were significant differences in the dietary habits between cases and controls. Lifestyles, such as smoking, alcohol consumption and tea consumption, of the two groups were also significantly different (table 2). 
Table 1 Single conditional logistic regression analysis on dietary factors, $\mathrm{n}(\%)$

\begin{tabular}{|c|c|c|c|c|c|c|}
\hline Factor & Frequency* & Cases & Control & $P$ value & OR & $95 \% \mathrm{Cl}$ \\
\hline \multirow[t]{3}{*}{ Breakfast consumption } & + & $112(62.22)$ & $153(85.00)$ & & 1.00 & \\
\hline & ++ & $38(21.11)$ & $18(10.00)$ & $<0.01$ & 2.84 & $1.54-5.25$ \\
\hline & +++ & $30(16.67)$ & $9(5.00)$ & $<0.01$ & 4.78 & $2.10-10.87$ \\
\hline \multirow[t]{3}{*}{ Meal duration } & $\geq 20$ min & $24(13.33)$ & $46(25.56)$ & & 1.00 & \\
\hline & $10-20 \min$ & $50(27.78)$ & 70 (38.89) & 0.393 & 1.32 & $0.70-2.52$ \\
\hline & $<20 \min$ & $106(58.89)$ & 64 (35.56) & $<0.01$ & 2.97 & $1.63-5.40$ \\
\hline \multirow[t]{3}{*}{ Onion or garlic } & + & $73(40.56)$ & 49 (27.22) & & 1.00 & \\
\hline & ++ & $66(36.67)$ & $56(31.11)$ & 0.29 & 0.74 & $0.42-1.30$ \\
\hline & +++ & $41(22.78)$ & $75(41.67)$ & $<0.01$ & 0.36 & $0.21-0.63$ \\
\hline \multirow[t]{3}{*}{ Spicy food } & + & $104(57.78)$ & $125(69.44)$ & & 1.00 & \\
\hline & ++ & $45(25.00)$ & $48(26.67)$ & 0.71 & 1.11 & $0.64-1.93$ \\
\hline & +++ & $31(17.22)$ & $7(3.89)$ & $<0.01$ & 5.95 & $2.28-15.53$ \\
\hline \multirow[t]{3}{*}{ Hot food } & + & $76(42.22)$ & $114(63.33)$ & & 1.00 & \\
\hline & ++ & $47(26.11)$ & $47(26.11)$ & 0.25 & 1.35 & $0.81-2.27$ \\
\hline & +++ & $57(31.67)$ & $19(10.56)$ & $<0.01$ & 5.63 & $2.72-11.66$ \\
\hline \multirow[t]{3}{*}{ Green vegetables } & + & $35(19.44)$ & $15(8.33)$ & & 1.00 & \\
\hline & ++ & 70 (388.9) & $46(25.56)$ & 0.34 & 0.71 & $0.36-1.43$ \\
\hline & +++ & $75(41.67)$ & $119(66.11)$ & $<0.01$ & 0.29 & $0.15-0.56$ \\
\hline \multirow[t]{3}{*}{ Fresh fruit } & + & $115(63.89)$ & $43(23.89)$ & & 1.00 & \\
\hline & ++ & $42(23.33)$ & $78(43.33)$ & $<0.01$ & 0.20 & $0.11-0.37$ \\
\hline & +++ & $23(12.78)$ & $59(32.78)$ & $<0.01$ & 0.16 & $0.08-0.32$ \\
\hline \multirow[t]{3}{*}{ Dairy } & + & $133(73.89)$ & $82(45.56)$ & & 1.00 & \\
\hline & ++ & $32(17.78)$ & $53(29.44)$ & $<0.01$ & 0.28 & $0.15-0.53$ \\
\hline & +++ & $15(8.33)$ & $45(25.00)$ & $<0.01$ & 0.17 & $0.08-0.36$ \\
\hline \multirow[t]{3}{*}{ Pickled vegetables } & + & $55(30.56)$ & $125(69.44)$ & & 1.00 & \\
\hline & ++ & $65(36.11)$ & $45(25.00)$ & $<0.01$ & 2.67 & $1.60-4.49$ \\
\hline & +++ & $60(33.33)$ & $10(5.56)$ & $<0.01$ & 15.27 & $5.91-39.49$ \\
\hline \multirow[t]{3}{*}{ Fish sauce } & + & $109(60.56)$ & $135(75.00)$ & & 1.00 & \\
\hline & ++ & $30(16.67)$ & $32(17.78)$ & 0.96 & 1.02 & $0.56-1.83$ \\
\hline & +++ & $41(22.78)$ & $13(7.22)$ & $<0.01$ & 3.81 & $1.89-7.65$ \\
\hline
\end{tabular}

*'+', never or seldom consuming; '++', sometimes consuming; '+++', regularly consuming.

Personality types, adaptability, feelings about life, irritability and interpersonal skills were also significantly different between cases and controls (table 2).

\section{Serum PG I, PG II, PG I/II ratio and G-17 levels}

Serum PG I, PG II and G-17 levels did not fit normal distributions, nor did the PG I/II ratio; therefore median values were used to indicate central tendencies and the P25 and P75 percentiles were used to indicate dispersion tendencies. Serum PG II and G-17 levels in patients with gastric cancer were higher than those in the control subjects $(p<0.05)$, while the PG I/II ratio was lower in the patients $(\mathrm{p}<0.05)$; PG I levels were not statistically different between the two groups. The results of the serum markers are summarised in table 3 .

\section{H. pylori infection}

As shown in table $4,66.67 \%$ of patients with gastric cancer were $H$. pylori positive, which was significantly higher than that of the control group $(54.44 \%, \mathrm{p}<0.05)$. The positive rate of $H$. pylori was higher in male patients with gastric cancer than in controls $(\mathrm{p}<0.05)$, however no significant differences were found between female cases and controls or among age groups $(\mathrm{p}>0.05)$.

\section{Serological parameters with different locations of gastric cancer}

Serum G-17 levels were higher in patients with gastric corpus cancer than in patients with gastric antrum cancer $(\mathrm{p}<0.05)$, but not significantly different among patients with other tumour locations ( $>0.05$; table 5 ). Serum PG 
Table 2 Single conditional logistic regression analysis on lifestyle habits and personality factors, $\mathrm{n}(\%)$

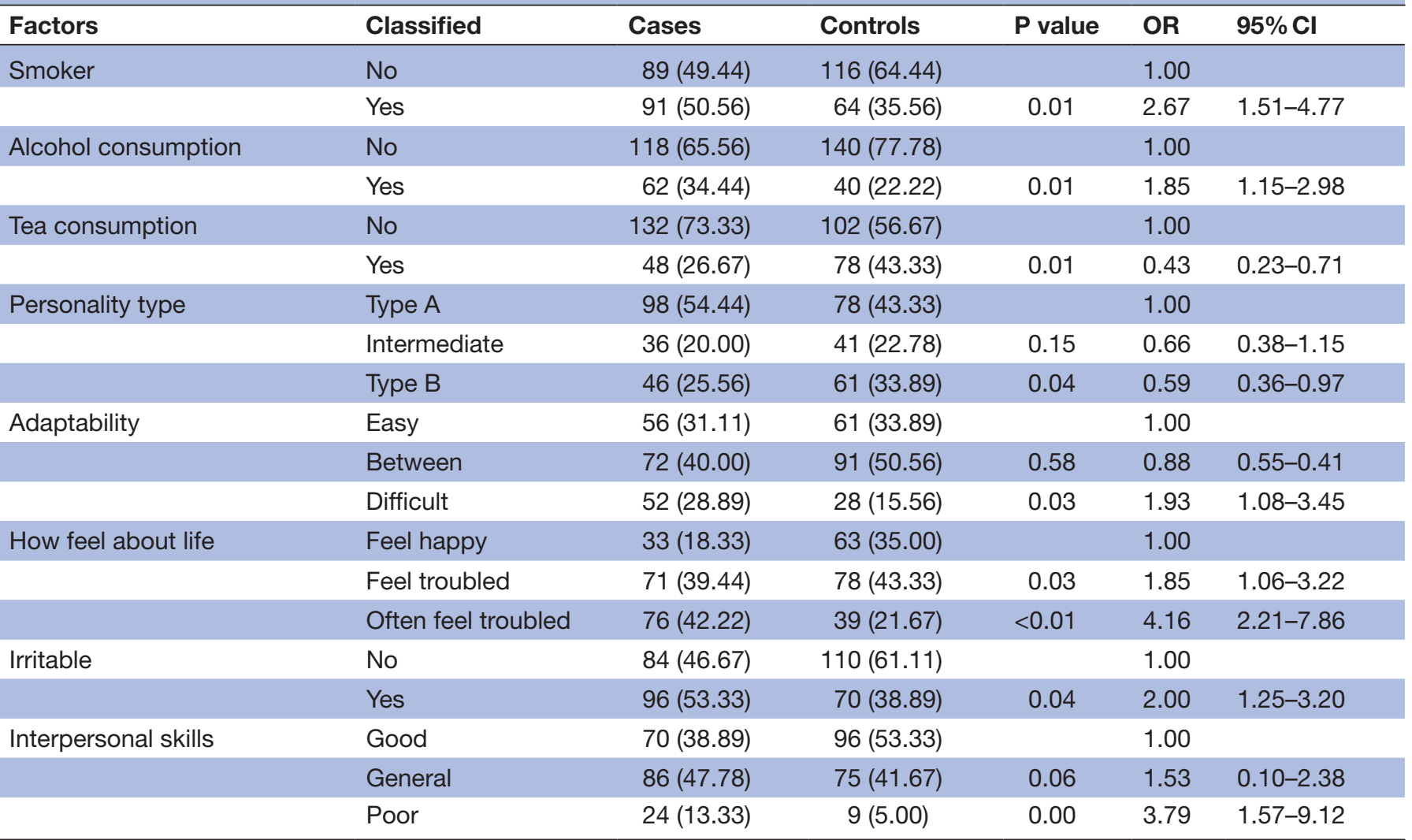

I, PG II levels and the PG I/II ratio were not statistically different among patients with different tumour locations ( $>0.05$; table 5).

\section{Serological parameters with different clinical stages of gastric cancer}

As shown in table 6, serum PG II levels in patients with advanced gastric cancer were higher than in patients with early-stage gastric cancer $(\mathrm{p}<0.05)$. However, the PG I/II ratio was lower in patients with advanced cancer than in patients with early-stage disease $(p<0.05)$. Serum PG I and G-17 levels were not significantly different between the two different clinical stages $(\mathrm{p}>0.05)$.

\section{Multivariate analysis}

Multivariate conditional logistic regression analysis was performed with gastric cancer as the independent variable, and the dietary/lifestyle habits, psychological factors, serum PG I level, serum PG II level, PG I/II ratio, serum G-17 level and $H$. pylori infection as dependent variables. As shown in table 7, eating hot food, consumption of pickled vegetables and often feel troubled may be risk factors for gastric cancer. However, the consumption of fresh fruit, onion or garlic, drinking tea, and elevated serum PG I levels and PG I/II ratio might be protective factors for gastric cancer. Other factors examined in this study had no statistically significant effect on gastric cancer $(\mathrm{p}>0.05)$.

\section{DISCUSSION}

Overall, this study has found that gastric cancer was related to several dietary and lifestyle factors, the changes in serum PG I/II ratio and G-17, and the infection rate of $H$. pylori. 
Table 4 Comparison of the Helicobacter pylori positive rates between cases and controls, $\mathrm{n}(\%)$

\begin{tabular}{lccll}
\hline Type & Cases & Controls & $\chi^{2}$ & P value \\
\hline $\begin{array}{l}\text { Total } \\
\text { Gender }\end{array}$ & $120(66.67)$ & $98(54.44)$ & 5.63 & 0.02 \\
$\quad$ Male & $94(52.22)$ & $77(42.78)$ & 4.88 & 0.03 \\
$\quad$ Female & $26(14.44)$ & $21(11.67)$ & 0.97 & 0.33 \\
$\chi^{2}$ & 2.65 & 1.20 & & \\
P value & 0.10 & 0.27 & & \\
Age (years) & & & & \\
$\quad<50$ & $20(11.11)$ & $16(8.89)$ & 0.86 & 0.36 \\
$50-65$ & $51(28.33)$ & $42(23.33)$ & 3.16 & 0.08 \\
$\quad \geq 65$ & $49(27.22)$ & $40(22.22)$ & 1.57 & 0.21 \\
$\chi^{2}$ & 0.55 & 1.28 & & \\
$P$ value & 0.76 & 0.53 & & \\
\hline
\end{tabular}

Multivariate conditional logistic regression analysis indicated that the consumption of pickled vegetables may be a risk factor for gastric cancer. Pickles are high in salt and may damage the gastric mucosa, reduce gastric acid secretion and inhibit the synthesis of prostaglandin E, which enhances gastric mucosa resistance. ${ }^{11}$ In addition, pickles contain high amount of nitrate and nitrite, which can be converted to N-nitrosamide, a carcinogen, under gastric acid conditions. ${ }^{12}$ In an area with high incidence of gastric cancer in northwest China's Gansu Province, ${ }^{13}$ where people often consume pickled vegetables as substitutions of fresh vegetables in winters, $65 \%-75 \%$ of pickles had detectable levels of nitrite and all had tested positive for nitrate; and the levels of nitrite and nitrate in the gastric juice were significantly higher in people who often consume pickled vegetables than in people who seldom consume pickled vegetables. Nitrate and nitrite levels in the gastric juice were associated with the frequency of pickle intake, which in turn was associated with gastric cancer. This study also found that regular pickle intake (seven or more times per week) increased the risk of gastric cancer $(\mathrm{OR}=4.05 ; 95 \%$ CI: 2.01-8.02).
Study results also indicated that tea consumption was a protective factor against gastric cancer. Tie Guanyin, one of the most popular teas in Fujian, is rich in tea polyphenols. Tea polyphenols can act as strong antioxidants to effectively remove free radicals. They can also regulate carcinogen-metabolising enzymes, inhibit the nitrosation reactions, block the expression of tumour genes, enhance immunity and therefore act as potent anticancer agents. ${ }^{14}$ However, a meta-analysis of 12 cohort studies from China, Japan and America suggested that drinking green tea $(\geq 5$ cups per day) was not correlated with the incidence of gastric cancer $(p>0.05) .{ }^{15}$ Stratified sex analyses showed that drinking $\geq 5$ cups of green tea per day was a protective factor against gastric cancer in women, but not in men. ${ }^{15}$ In this study, results showed that drinking tea lowered the risk of gastric cancer (OR=0.26, 95\% CI: $0.10-0.67)$, confirming the results shown by Mu et al. ${ }^{16}$ Our findings also indicated that drinking tea may be an independent protective factor against gastric cancer, however other factors, such as the type of tea and the water temperature, might affect this relationship and warrant further investigation.

As the medical model shifts from 'biomedical' to 'bio-psycho-social' paradigm, the relationship between psychological factors and tumour gradually catches the attention of numerous scholars in recent years. A metaanalysis including 5265 cases of gastric cancer and 12539 controls from 22 domestic studies found that long-term stress had adverse effects on gastric cancer (merged OR of 2.91, 95\% CI: 2.03-4.19) ${ }^{17}$ Another study showed that psychological factors were associated with the incidence of gastric cancer and that history of metal stimulus increased the risk of gastric cancer significantly $(\mathrm{OR}=1.74,95 \% \mathrm{CI}$ : 1.11-2.74) ${ }^{18}$ People who often feel anxious/irritable or experience hardships in life have altered hormone levels, which in turn affect their immune system. In particular, epinephrine and norepinephrine are released under stress or anxiety, causing decrease in natural killer cells, reduction in immunity functions and acceleration in the initiation and progression of malignancies. ${ }^{19}$ Similar to previous studies, this study also found that often feel troubled was related to gastric cancer $(\mathrm{OR}=2.21,95 \% \mathrm{CI}$ : 1.23-3.98) in our subjects.

\begin{tabular}{|c|c|c|c|c|c|}
\hline Location & $\begin{array}{l}\text { Cases } \\
\text { (n) }\end{array}$ & PG I ( $\mu \mathrm{g} / \mathrm{L})$ & PG II ( $\mu g / L)$ & PG I/II ratio & Gastrin-17 (pmol/L) \\
\hline Cardia & 19 & $116.15(61.42,182.66)$ & $17.27(11.74,28.83)$ & $6.41(5.09,8.01)$ & $6.35(2.36,10.75)$ \\
\hline Fundus & 17 & $81.26(53.44,197.57)$ & $20.72(9.32,33.06)$ & $5.22(3.29,8.41)$ & $8.14(3.85,12.50)$ \\
\hline Gastric corpus & 55 & $118.23(66.41,175.04)$ & $19.43(12.25,28.94)$ & $5.69(3.78,8.23)$ & $12.00(6.58,19.74)$ \\
\hline Gastric antrum & 70 & $118.65(78.87,173.66)$ & $16.79(10.42,27.49)$ & $7.05(5.45,9.07)$ & $6.12(3.84, \mathrm{t} 12.67)$ \\
\hline Gastric angle & 19 & $116.00(68.13,195.76)$ & $15.89(7.39,29.28)$ & $7.25(5.63,8.10)$ & $6.44(2.58,4.81)$ \\
\hline$\chi^{2}$ & & 1.58 & 1.59 & 7.41 & 10.79 \\
\hline$P$ value & & 0.81 & 0.81 & 0.12 & 0.03 \\
\hline
\end{tabular}

$P G$, pepsinogen. 
Table 6 Comparison of the serological parameters in patients with different clinical stages of gastric cancer, M (P25, P75)

\begin{tabular}{llllll}
\hline Clinical stage & $\begin{array}{l}\text { Cases } \\
(\mathbf{n})\end{array}$ & PG I $(\boldsymbol{\mu g} / \mathbf{L})$ & PG II ( $\mathbf{\mu g} / \mathbf{L})$ & PG I/II ratio & Gastrin-17 (pmol/L) \\
\hline Early & 49 & $93.07(68.39,171.64)$ & $13.81(7.42,23.92)$ & $6.95(5.71,9.73)$ & $5.93(2.85,12.75)$ \\
Advanced & 131 & $118.23(69.43,181.24)$ & $19.43(12.51,30.41)$ & $6.10(4.50,8.03)$ & $8.19(4.29,14.39)$ \\
$\quad$ Z & & 0.88 & 2.33 & 2.43 & 1.75 \\
$\quad$ P value & & 0.38 & 0.02 & 0.02 & 0.08 \\
\hline
\end{tabular}

PG, pepsinogen.

Recent studies indicated that low serum PG I and PG I/ II ratio were strongly associated with gastric cancer, while the relationship between PG II and gastric cancer was not as obvious. PG I is primarily secreted by chief cells and mucous neck cells in the fundic glands, whereas PG II is secreted by all gastric glands and the proximal duodenal mucosa. ${ }^{20}$ When chronic $H$. pylori infection with chronic atrophic gastritis (CAG) extends from antrum to corpus of stomach, chief cells are replaced by pyloric glands. Therefore, the concentration of serum PG I will decrease due to damaged secretion ability of gastric mucosa, however the secretion of PG II remains, resulting in a lowered PG I/ II ratio, which would reflect the severity of CAG. Patients with premalignant lesions, such as CAG or dysplasia, have considerable higher risks for developing gastric cancer. Our previous study ${ }^{21}$ explored the changes of serum PG levels in different gastric cancer states. Results found significant differences in serum PG I, PG II and PG I/ II ratio among the control group, the atrophic gastritis group and the gastric cancer group (all $\mathrm{p}<0.001)$. Serum PG I level was lower in the gastric cancer group than in the other two groups, and was also lower in the atrophic gastritis group than in the control group, both differences were statistically significant $(\mathrm{p}<0.05)$. Serum PG I/II ratio in the gastric cancer group was lower than in the control group and the atrophic gastritis group, and was also lower in the atrophic gastritis group than in the control group, both differences were statistically significant $(p<0.05)$. In a study in Japan including 10996 healthy residents who had undergone gastroscope examinations and tested for serum PG, the incidence of gastric cancer was higher in subjects with lower serum PG I levels and PG I/II ratios. ${ }^{22}$ In our study, single conditional logistic regression results showed that serum PG II was higher in patients with gastric cancer than in control subjects $(\mathrm{p}<0.05), \mathrm{PG}$ I/ II ratio was lower in patients with gastric cancer than in control subjects $(\mathrm{p}<0.05)$ and PG I levels in the two groups were not significantly different $(p>0.05)$, which was in agreement with the study conducted by Cao $e t a l^{23}$ Moreover, our results showed that after controlling for other risk factors, decreased levels of serum PG I and PG I/II ratios might be independent risk factors for gastric cancer, which was in agreement with the results of a nested case-control study conducted by Kurilovich et al. ${ }^{24}$

Qin $e t a l^{25}$ found that serum PG I and PG II levels were lower in patients with advanced-stage gastric cancer than in those with early-stage disease $(p<0.05)$, which was consistent with the results observed in the elderly patients with gastric cancer (aged $>60$ years) by Wei $e t$ $a l^{26}$ In this study, serum PG II levels were significantly higher in patients with advanced-stage gastric cancer than those with early-stage disease $(\mathrm{p}<0.05)$, PG I/II ratio was significantly lower in patients with advanced-stage gastric cancer $(\mathrm{p}<0.05)$. These results demonstrated that serum PG II level and PG I/II ratio were different at different clinical stages of gastric cancer. Therefore, the elevation of serum PG II levels and reduced PG I/II ratio may be indicators of the clinical stages of gastric cancer.

Gastrin, a peptide hormone synthesised and secreted by the $\mathrm{G}$ cells of the pyloric antrum of the stomach, was

Table 7 Multivariate conditional logistic regression analysis

\begin{tabular}{lccrccc}
\hline Factors & $\boldsymbol{\beta}$ & SE & Wald & P value & OR & $\mathbf{9 5 \%} \mathbf{C l}$ \\
\hline Hot food & 0.84 & 0.31 & 7.63 & 0.006 & 2.32 & $1.28-4.23$ \\
Onion or garlic & -1.05 & 0.33 & 10.11 & 0.001 & 0.35 & $0.18-0.67$ \\
Fresh fruit & -0.60 & 0.29 & 4.34 & 0.037 & 0.55 & $0.31-0.97$ \\
Pickled vegetables & 1.39 & 0.35 & 15.44 & $<0.001$ & 4.05 & $2.01-8.03$ \\
Tea consumption & -1.35 & 0.49 & 7.77 & 0.005 & 0.26 & $0.10-0.67$ \\
Often feel troubled & 0.79 & 0.30 & 7.03 & 0.008 & 2.21 & $1.23-3.98$ \\
PG I & -0.01 & 0.00 & 3.96 & 0.047 & 0.99 & $0.99-1.00$ \\
PG I/II ratio & -0.32 & 0.07 & 20.82 & $<0.001$ & 0.73 & $0.63-0.83$ \\
\hline
\end{tabular}

$P G$, pepsinogen. 
first discovered in $1906,{ }^{27}$ and is now well known for its multiple subtypes. ${ }^{28}$ Ninety per cent of the intracorporeal gastrin is G-17, which is one of the main forms in gastrin circulation, and the change of which can indicate the impairment of gastric mucosa functions. ${ }^{29} \mathrm{Li} e t$ $a l^{30}$ had used immunohistochemical method to detect serum G-17, and found it had increased concentrations in superficial gastritis, paracancerous tissues and gastric cancer cells. In addition, serum G-17 levels were found to be higher in patients with preoperative gastric cancer than in healthy controls $(\mathrm{p}<0.05)$, and were also elevated in patients with advanced clinical stage of gastric cancer. ${ }^{31}$

Our finding was coincided with the study conducted by Rui et al, that serum G-17 in patients with gastric cancer was higher than in the controls $(\mathrm{p}<0.05) .{ }^{32}$ This indicated that increased serum G-17 levels were associated with gastric cancer and hypergastrinaemia. In terms of the potential mechanism of action, animal experiments with overexpressed G-17 indicated that gastrin could influence gastric carcinogenesis. ${ }^{33}$ Serum G-17 levels were found to be higher in patients with gastric corpus cancer than in patients with gastric cancer in other locations $(p<0.05)$, which was similar to the findings described by Hua et al. ${ }^{34}$ This may be related to the vagus nerve's depressive effect on G-17 secretion. Serum G-17 levels were more clearly elevated when cancer invaded the gastric body, which could damage the vagus nerve and inhibit G-17 secretion. ${ }^{35}$ Therefore, our results suggested that changes in serum G-17 levels in patients with gastric cancer may be indicative of cancer location.

H. pylori infection was a risk factor for gastric cancer. The International Agency for Research on Cancer formally identified $H$. pylori as class I carcinogen in 1994. In addition, a 7.8-year prospective study of 1526 Japanese patients with gastric diseases showed that patients with $H$. pylori infection had diseases such as gastric atrophy, intestinal metaplasia, as well as precancerous lesions. ${ }^{36}$ Moreover, a study conducted in Changle City, Fujian Province, China, showed that although patients with precancerous gastric lesions were unable to avoid gastric cancer, $H$. pylori eradication could reduce the risk of gastric cancer in people without precancerous lesions. ${ }^{37}$ The H. pylori infection rate in the control group in the current study was $54.4 \%$, which was close to the number found in natural population $(54.76 \%) .{ }^{38}$ However, the infection rate in patients with gastric cancer was $66.7 \%$, similar to the results observed by Xueyuan $e t a l^{39}$ suggesting that $H$. pylori infection was more common in patients with gastric cancer. In addition, the $H$. pylori infection rate in the gastric cancer group was significantly higher than in the control group in male subjects $(p<0.05)$, whereas no such difference was observed in women, which might be associated with a lower prevalence of $H$. pylori infection in women. ${ }^{40}$

There were some strengths in this study. First, Fujian Province is an area with high incidence of gastric cancer in China. According to the report of Fujian Province, the incidence of gastric cancer collected from tumour registration areas in Fujian Province in 2017 was 31.68/100 000, accounting for $12.47 \%$ of all new cancer cases, ranking third in cancer incidence; gastric cancer mortality rate was $25.90 / 100000$, accounting for $14.74 \%$ of all cancer deaths, also ranking third in cancer deaths. Therefore, Fujian is an important research site for exploring the causes of gastric cancer. Second, strict quality control was conducted in this study in the selection of new cases to make sure the results be comparable. Taking into account the effects of proton pump inhibitors and $\mathrm{H}$ receptor antagonists on serum markers, patients who had taken these drugs within a week before recruiting were excluded. With strictly controlling the quality of selected cases, the difficulty in obtaining cases also increased. Third, this study was one of the few studies to use serum indicators as independent variables to explore the risk factors for gastric cancer. However, several limitations should be considered. First, for a case-control study, the causal association between dietary/lifestyle habits and gastric cancer could not be precisely identified. Second, the consumptions of alcohol, tea and pickled vegetables were self-reported. Subjects often had difficulties in recalling food consumptions and it was also hard to estimate the accurate amount of consumption. Therefore, recall bias and misclassification bias were inevitable. Randomised control trial studies shall be conducted for more accurate results. Third, the sample size was relatively small. China has high incidence of gastric cancer and provides favourable conditions for studying it. We plan to expand the sample size in our further researches on gastric cancer.

\section{Conclusion}

In conclusion, this study indicated that serum PG and G-17 levels, as well as the detection of $H$. pylori antibodies, could be useful indicators of gastric cancer location and cancer stage. Elevation of serum G-17 levels may be indicative of the gastric cancer location, while the increase in the PG II level and the reduction in the PG I/II ratio may imply the clinical stage. Poor dietary habits, salty food and often feel troubled may be risk factors for gastric cancer, while eating fresh fruit, onion or garlic, and drinking tea may help protect against this disease.

\section{Author affiliations}

${ }^{1}$ Fujian Key Laboratory of Zoonoses Research, Fujian Center for Disease Control and Prevention, Fuzhou, Fujian, China

${ }^{2}$ School of Public Health, Fujian Medical University, Fuzhou, Fujian, China ${ }^{3}$ Chronic Disease Prevention, Fujian Center for Disease Control and Prevention, Fuzhou, Fujian, China

${ }^{4}$ Gastrointestinal Surgery, Fujian Provincial Hospital, Fuzhou, Fujian, China ${ }^{5}$ Gastroenterology, Fuzhou General Hospital of Nanjing Military Command Area, Fuzhou, Fujian, China

Acknowledgements We would like to thank Kazuo Aoki for co-designing the study and Zheng Huang for proofreading this manuscript. We also appreciate the assistance of the hospital staff in collecting blood samples.

Contributors PY and LL are joint first authors. KZ and WW obtained funding. KZ designed the study. LL drafted the manuscript. PY contributed to the interpretation of the results and critical revision of the manuscript for important intellectual 
content. WW, SW and LH collected the data. BW and TC detected serological indicators. XL analysed the data. All authors have read and approved the final manuscript. KZ and LC are the study guarantors.

Funding This work was supported by National Health and Family Planning Commission Research Fund (No. WKJ-FJ-18), Fujian Science and Technology Innovation Joint Fund Project (No. 2019Y9103) and National Key Research and Development Program 'Precision Medicine Research' (No. 2017YFC0908300).

Competing interests None declared.

Patient and public involvement Patients and/or the public were involved in the design, or conduct, or reporting, or dissemination plans of this research. Refer to the Methods section for further details.

Patient consent for publication Not required.

Ethics approval This study was approved by the ethical reviews committee at Fujian Center for Disease Control and Prevention.

Provenance and peer review Not commissioned; externally peer reviewed.

Data availability statement Data are stored in Fujian Center for Disease Control and Prevention, No. 76 Jintai Road, Gulou District, Fuzhou, China and are available upon reasonable request. Please sent Email to Kui-cheng Zheng at zkcfjcdc@sina. com.

Open access This is an open access article distributed in accordance with the Creative Commons Attribution Non Commercial (CC BY-NC 4.0) license, which permits others to distribute, remix, adapt, build upon this work non-commercially, and license their derivative works on different terms, provided the original work is properly cited, appropriate credit is given, any changes made indicated, and the use is non-commercial. See: http://creativecommons.org/licenses/by-nc/4.0/.

\section{ORCID iD}

Ping Yuan http://orcid.org/0000-0002-9027-4499

\section{REFERENCES}

1 Bray F, Ferlay J, Soerjomataram I, et al. Global cancer statistics 2018: GLOBOCAN estimates of incidence and mortality worldwide for 36 cancers in 185 countries. CA Cancer J Clin 2018;68:394-424.

2 Zhou M, Wang H, Zhu J, et al. Cause-Specific mortality for 240 causes in China during 1990-2013: a systematic subnational analysis for the global burden of disease study 2013. Lancet 2016;387:251-72.

3 Feng R-M, Zong Y-N, Cao S-M, et al. Current cancer situation in China: good or bad news from the 2018 global cancer statistics? Cancer Commun 2019;39:22.

4 Xiongwei Z. 2017 Fujian cancer registry annual report. Fujian Science and Technology Press, 2018.

5 Cheng XJ, Lin JC, Tu SP. Etiology and prevention of gastric cancer. Gastrointest Tumors 2016;3:25-36.

6 Venerito M, Vasapolli R, Rokkas T, et al. Gastric cancer: epidemiology, prevention, and therapy. Helicobacter 2018;23 Suppl 1:e12518.

7 You WC, Li JY, Zhang L, et al. Etiology and prevention of gastric cancer: a population study in a high risk area of China. Chin J Dig Dis 2005;6:149-54.

8 You WC, Li JY, Zhang L, et al. Etiology and prevention of gastric cancer in Linqu County, Shandong Province, China. Chin J Dig Dis 2005;6:112-3.

9 Zabaleta J. Multifactorial etiology of gastric cancer. Methods Mol Biol 2012;863:411-35

10 Zhang G, Li J, Li S, et al. Exploring spatial trends and influencing factors for gastric cancer based on Bayesian statistics: a case study of Shanxi, China. Int J Environ Res Public Health 2018;15. doi:10.3390/ijerph15091824. [Epub ahead of print: 23 Aug 2018].

11 Karagulle M, Fidan E, Kavgaci $\mathrm{H}$, et al. The effects of environmental and dietary factors on the development of gastric cancer. J Buon 2014;19:1076-82.

12 Kaifeng P, Deng D, Li T. Preliminary study on microorganism participation and synthesis of Nitrosamides in gastric juice. Chinese Journal of Preventive 1995;29:222-4.

13 Yumin L, Chen L, Xue Q. Measure of nitro-compounds in pickles and analysis of causes for high incidence of gastric cancer in Wuwei area. Analysis of Testing Technology and Instruments 2003;9:88-90.

14 Zongheng L. Healthy Fuction of Tieguanyin. Tea in Fujian 2010;32:44-8.
15 Jiwu G, Li B, Wang T. Meta analysis of cohort study on green tea and gastric cancer risk. J Third Mil Med Univ 2014;36:1914-9.

$16 \mathrm{Mu}$ L. Study on risk factors, protective factors and molecular epidemiology on three upper Gi cancers in Taixing area. Doctoral Dissertation from Fudan University, 2002: 45-55.

17 Yumin G, Hu Y, Xin Z. Meta Analysis of Relationship among Psychological Factors, Alcohol \& Tobacco \& Tea Hobby and Gastric Cancer. Inner Mongolia Medical Journal 2005;27:284-6.

18 Xiangdong J, Dai L, Zhang J. Case-Control family study on risk factors of gastric cancer in rural areas of Henan Province. Journal of Central South University 2007;32:782-4.

19 Xibin S, Lu J, Dai D. Case-Control study of relationship among psychological factors, cancer family history and gastric cancer. Chronic Disease Prevention and Control in China 2001;9:12-14.

20 Bang CS, Lee JJ, Baik GH. Prediction of chronic atrophic gastritis and gastric neoplasms by serum pepsinogen assay: a systematic review and meta-analysis of diagnostic test accuracy. J Clin Med 2019;8. doi:10.3390/jcm8050657. [Epub ahead of print: 10 May 2019].

21 Wu S. Study on the cut-off value of serological markers in screening gastric cancer atrophic gastritis and related factors in residents with low and high incidence of gastric cancer in Fujian Province. Fujian Medical University, 2017.

22 Yoshihara M, Sumii K, Haruma K, et al. Correlation of ratio of serum pepsinogen I and II with prevalence of gastric cancer and adenoma in Japanese subjects. Am J Gastroenterol 1998;93:1090-6.

23 Cao X-Y, Jia Z-F, Jin M-S, et al. Serum pepsinogen II is a better diagnostic marker in gastric cancer. World J Gastroenterol 2012;18:7357-61.

24 Kurilovich S, Belkovets A, Reshetnikov O, et al. Stomach-specific biomarkers (GastroPanel) can predict the development of gastric cancer in a Caucasian population: a longitudinal nested case-control study in Siberia. Anticancer Res 2016;36:247-53.

25 Qin C, Ran Z, Xiao S. Diagnosis of clinical values for gastric cancer by detecting serum pepsinogen and gastrin-17. J Gastroenterol Hepatol 2007;16:361-4

26 Wei H, Cai M, Wu Y. Clinical significance of combination detection with serum pepsinogen and gastrin-17 in senile gastric cancers and atrophic gastritis. Guide of China Medicine 2008;6:134-6.

27 Edkins JS. The chemical mechanism of gastric secretion. J Physiol 1906;34:133-44.

28 Juan D, Wang X. Bioactivity and signal transduction pathway of gastrin. Progress of Anatomical Sciences 2003;9:347-52.

29 Shulkes A, Baldwin G. Biology and pathology of non-amidated gastrins. Scand J Clin Lab Invest Suppl 2001;234:123-8.

30 Li W, Zhou L, Li Y. Expression differences and significance of gastrin in gastritis and gastric cancers. Chinese Journal of Minimally Invasive Surgery 2011;11:435-8.

31 TianHu L. Clinical analysis of detection in REGARDS to serum gas, IGF-I levels for patients with gastric cancers before and after operation. China Medical Herald 2011;8:13-14.

32 Rui L, Ren H, Tian L. Study on the lever of serum PG and gastrin for H.pylori infection positive patients with gastric cancer. Chinese Journal of Laboratory Diagnosis 2015;11:1250-1.

33 Wang TC, Dangler CA, Chen D, et al. Synergistic interaction between hypergastrinemia and Helicobacter infection in a mouse model of gastric cancer. Gastroenterology 2000;118:36-47.

$34 \mathrm{Hua} \mathrm{H}$, Jin J. The change and significance of serum survivin antibodies and gastrin levels for patients with gastric cancer. Shandong Medicine 2013;53:45-6.

35 Xingsong T, Gong D, Zhou W. Relationship between the change of serum gastrin, gastric cancer and its prognosis. Shandong Medicine 1988;38:1-3.

36 Zullo A, Hassan C, Morini S. Helicobacter pylori infection and the development of gastric cancer. N Engl J Med 2002;346:65-7.

37 Wong BC-Y, Lam SK, Wong WM, et al. Helicobacter pylori eradication to prevent gastric cancer in a high-risk region of China: a randomized controlled trial. JAMA 2004;291:187-94.

38 Wandai Z, Hu F, Xiao S. Epidemiological investigation of natural population with H.pylori infection in China. Modern Digestion \& Intervention 2010;15:265-70.

39 Xueyuan C, Jia Z. Correlation analysis of serum H.pylori lgG antibody, pepsinogen and gastric cancer incidence. China Laboratory Diagnosis 2012;6:1026-8.

40 Ibrahim A, Morais S, Ferro A, et al. Sex-differences in the prevalence of Helicobacter pylori infection in pediatric and adult populations: systematic review and meta-analysis of 244 studies. Dig Liver Dis 2017;49:742-9. 